\title{
HACMAC: A reliable Human ACtivity-based Medium Access Control for implantable body sensor networks
}

\author{
Vignesh Raja Karuppiah Ramachandran, Paul J.M. Havinga, Nirvana Meratnia \\ Pervasive Systems Research Group \\ Department of Computer Science \\ University of Twente, The Netherlands \\ Email: \{v.r.karuppiahramachandran, p.j.m.havinga, n.meratnia\}@utwente.nl
}

\begin{abstract}
Chronic care is an eminent application of implantable body sensor networks (IBSN). Performing physical activities such as walking, running, and sitting is unavoidable during the long-term monitoring of chronic-care patients. These physical activities cripple the radio frequency (RF) signal between the implanted sensor nodes. This is because various body postures shadow the RF signal. Although shadowing itself may be short, a prolonged activity will significantly increase the effect of the RF-shadowing. This effect dampens the communication between implantable sensor nodes and hence increases the chance of missing life-critical data. To overcome this problem, in this paper we propose a link quality-aware medium access control (MAC) protocol called HACMAC, which adapts the access mechanism during different human activities based on the wireless link-quality. Our simulation results show that compared with the access mechanism suggested by the IEEE 802.15.6 standard, the reliability of the wireless communication is increased using HACMAC even while transmitting at a strongly low transmission power of $25 \mu \mathrm{W}$ effective isotropic radiated power (EIRP) set by the IEEE 802.15.6 standard.
\end{abstract}

\section{INTRODUCTION}

Health-care industry is undergoing crucial changes as the common practice of clinical treatment is gradually being overhauled by ubiquitous health-care systems [1] [2] [3]. In the past decade, health-care organizations underwent steep rise of pressure to provide improved health-care as the number of chronic disease patients increases every year world wide [4]. Chronic diseases such as heart and lung diseases require real time, continuous, and long-term follow-ups. Energy-efficient medical sensors and devices, both wearable and implantable, are suitable for this purpose. However the therapeutic applications of these devices are still limited due to the inter-disciplinary challenges from different aspects such as bio-compatibility, size, chip design, and wireless communication [5] [6].

A network of these implantable medical devices is called the implantable body sensor networks (IBSN). IBSN is capable of improving the therapeutic significance of the longterm treatments by collective-diagnosis, in which different symptoms of the chronic care patients are continuously monitored using subcutaneously implanted sensors. This diagnostic information is used as a feedback to adapt the treatment delivered by medical devices such as pacemakers and deep brain simulators (DBS) in real-time, resulting in the closed-loop operation of medical devices. Intervention by physicians will be necessary only in the case of anomalies. For example, in the case of epileptic patients, physiological information from sensors such as Electroencephalogram (EEG) and Electrocardiogram (ECG) together with physical information from sensors such as accelerometers, sweat, and breathing can be used to predict the onset of epileptic seizures with better accuracy [7]. Once the onset of seizures is predicted, DBS should be activated with pre-stored patient specific parameters which can reduce the progression of the seizures in real-time. In case of any deviation from the known characteristics of patient-specific parameters, physicians are immediately alerted.

In the medical scenarios such as the one described above, wireless communication between the medical sensors and devices is inevitable. Human body is a conductive medium, which makes the wireless communication in and around the human body challenging. Although different wireless technologies such as ultra-sound and magnetic induction are emerging, RF communication is widely used because of the well established knowledge of the nano-sized design of RF components, bio-compatibility, and low power consumption of the hardware [5]. However, the propagation behavior of the RF signal in and around the human-body, reliable communication without data-loss, ultra-low power consumption are still among the open research topics.

Medium access control (MAC) protocol regulates the sensor node's access to the physical medium (e.g. the RF spectrum). MAC protocols are mainly engineered to improve the reliability of wireless communication by (i) reducing collision (i.e., two nodes accessing the medium at the same time) and the data loss caused by it, (ii) lowering energy consumption by reducing idle-listening (i.e., a node listening for datapackets while no data-packets are being sent), and (iii) overhearing (i.e., a node receives data-packets which are not destined for it). Advanced MAC protocols are designed to adapt to the quality of the physical medium. This adaptation means that communication is initiated only when quality of the RF link (calculated based on received signal strength information (RSSI)) between the sender and receiver is sufficient to successfully transmit and receive the data. Link quality estimation (LQE) is used to estimate whether the communication between the nodes will be successful. 


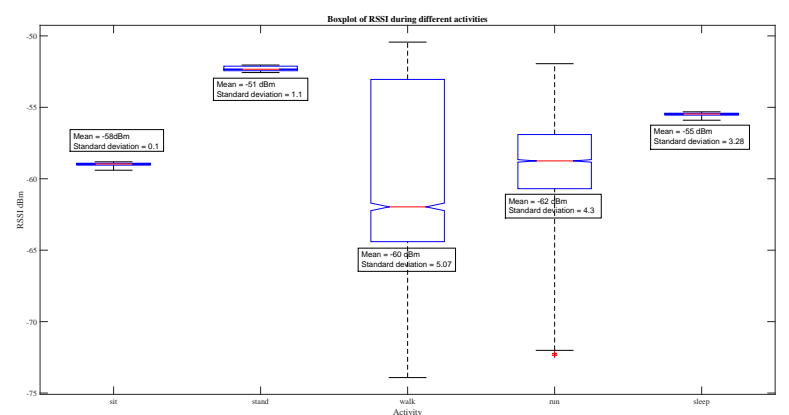

Fig. 1: Boxplot of RSSI data while performing different activities

LQE is a proprietary metric of Texas Instruments, which calculates the link quality at the chip by comparing the received frequency of bits with the expected frequency of bits according to the modulation schemes used by the chip. Other popular link quality metrics such, estimated number of transmission (ETX) [18], requested number of packets (RNP), and 4 bit (4B), use packet reception information to calculate the link quality.

Link quality between IBSN's sensor nodes suffer greatly from signal attenuation caused by simple human activities such as walking, running, sitting, and standing. Especially, the collective-diagnosis in chronic patients can be greatly affected due to the disruption in communication caused by the interference of the human activities in the RF link. Impact of human activities on the RF spectrum has been studied before and it has been shown that each activity has its unique pattern regarding influencing the RF spectrum [8].

\section{Motivation AND Related WORK}

In our previous work [12], we compared existing MAC protocols for the closed loop operation of medical devices and identified the potential drawbacks of MAC protocols. One of these drawbacks is for example the strong constraint of $25 \mu \mathrm{W}$ EIRP on the transmission power for active medical implants, which will cause the existing MAC protocols to fail in guaranteeing the required PDR. In this respect, reliability is the key feature of HACMAC, which is ensured through the following mechanisms:

- Hybrid access mechanism to support reliable transmission of high priority medical data.

- $\quad$ Modified IEEE 802.15.6 MAC protocol to support long term monitoring with medical implants.

- Adaptive scheduling.

- Fault tolerant link management to overcome RF shadowing effect while performing different activities.

Use of RSSI value to calculate the link quality is a common method in designing MAC protocols aiming to ensure high PDR [15] [9]. However, various approaches are used to estimate the link quality for different MAC protocols. For example in BANMAC [16], authors predict an opportunistic time window (OTW) by recording the RSSI values with a central controller in a network of 5 nodes. They achieve a high PDR of $96 \%$ at a very low transition power of $-21 \mathrm{dBm}$, simply by transmitting only during the predicted OTW. This achieved PDR is considerably high compared to the $73 \%$ of PDR achieved by the IEEE 802.15.4 MAC. Accurate estimation of the opportunistic time window (OTW) as done in BANMAC requires complex calculations, which require a powerful central controller such as mobile phones to be always connected to the network. Such complex calculations cannot be performed by computationally primitive nodes of IBSN. Also the external nodes in IBSN have less computational power than mobile phones and cannot support extremely-long (in terms of months) wearable periods between charging cycles. In BATMAC [17], authors used relay nodes to communicate between the transmitting node and the receiving node, when the wireless link between the sender and the receiver was shadowed by human body while performing different activities. BATMAC calculates the link quality using the PDR of the acknowledgement packets sent and received by the nodes, and forwards the packets through a relay if the calculated PDR is lower than the expected PDR. There are different methods to quantify the human activity from RSSI data, for example using bandpass filter followed by a fast fourier transformation (FFT) [9] to extract the human activity information from RSSI in the frequency domain. Less complex analysis in time domain and statistical domain also exist [10] [11], which are easier to be implemented on resource-constraint IBSN nodes.

In contrary to the existing works, we define the human activity in which the human is not only physically active (e.g. walking and running) but also idly active for example while sitting, sleeping, and standing. We measured RSSI data while performing different activities using the CC430 based on-body sensor nodes which is described in [12]. We made these measurements between two on-body sensor nodes placed on the ankle and on the waist, while the person was in an indoor-office environment. The measurements were made without MAC implementation and RSSI was recorded every $100 \mathrm{~ms}$, which was sufficiently longer to record the impact of the activities in RF signal. Transmission power was set to $-12 \mathrm{dBm}$ at the ankle-node and waistnode was receiving continuously (for details about hardware specifications refer [12]). Our initial study shows that even idle activities greatly influence the RF signal. The magnitude of attenuation caused by different activities can be seen from Figure 1, in which mean and standard deviation of the RSSI during different activities are illustrated. It can be seen that each activity has its own particular deviation with respect to the RSSI values. Additionally, activities such as sleeping, sitting, and standing have constant attenuation for long period of time which results in very low RSSI and hence low packet delivery ratio (PDR). Figure 2 shows distribution of RSSI and PDR while performing different activities. It can be understood from the figure how the RF signal is affected by different activities. A high PDR means a favorable link quality. The RSSI value is clipped in the range of $-65 \mathrm{dBm}$ and $-90 \mathrm{dBm}$ specifically to (i) show the effect of low RSSI and (ii) show that low RSSI does not always result in a low link quality. This is because the link quality also depends on the RF noise level and multi-path effect of the environment. Figure 2(e), shows a clear indication of walking pattern of a person walking at a speed of $4 \mathrm{~km} / \mathrm{h}$ with each step lasting about 1.2 seconds. It can be seen that at very low RSSI, PDR is still high for a short duration of time between each step indicating the line of sight between 

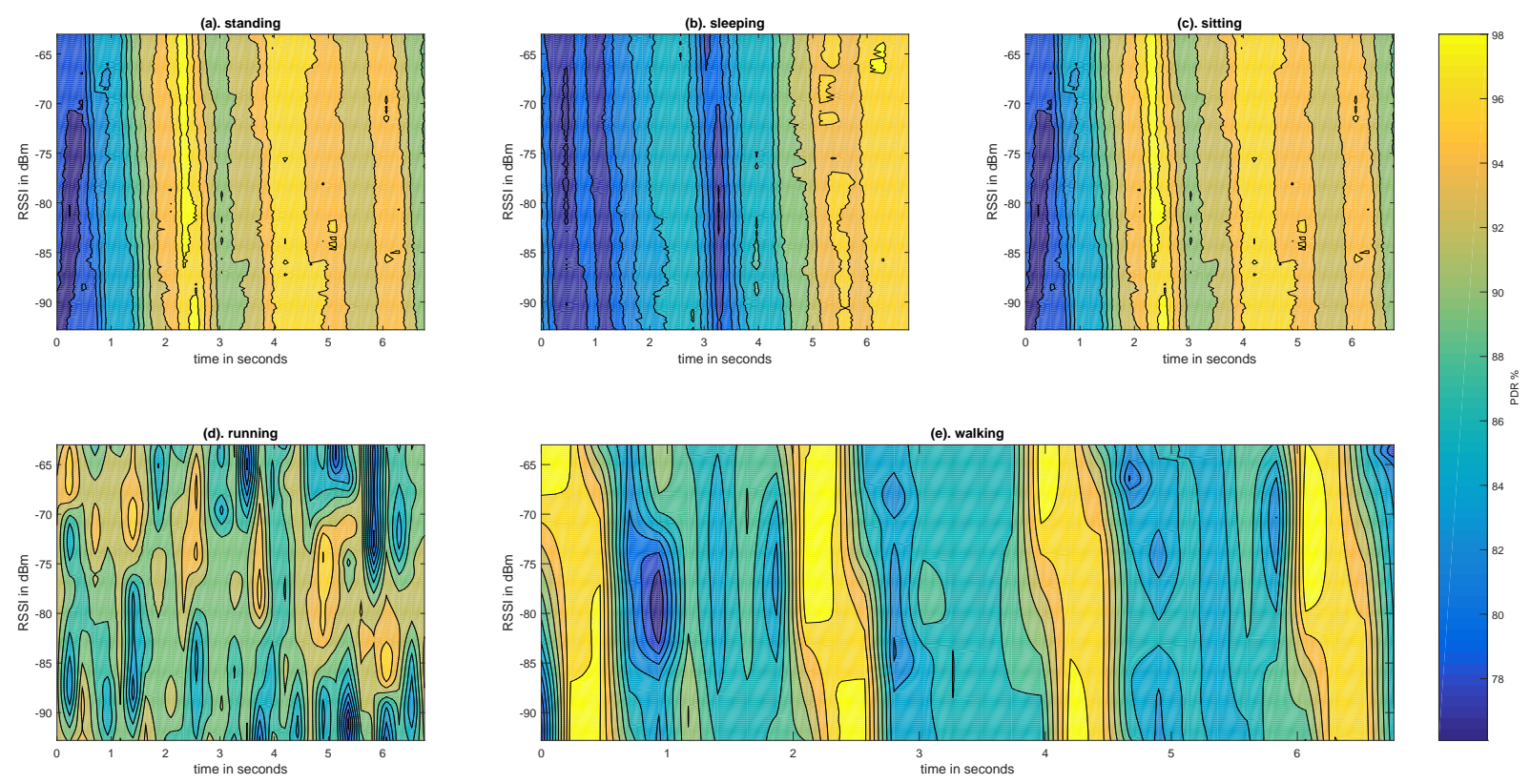

Fig. 2: Time vs RSSI. The colormap shows the distribution of PDR during each activity

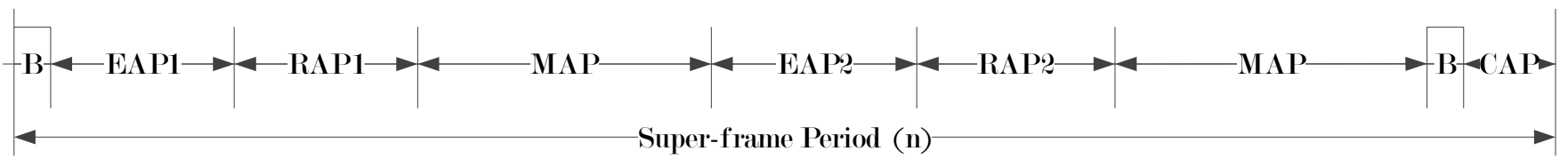

Fig. 3: Super-frame specified by the IEEE 802.15.6 standard [14]

ankle and waist nodes. Figure 2(d) shows that the running activity, in which the person is running at a speed of about $14 \mathrm{~km} / \mathrm{h}$ with each step lasting about 0.6 seconds. It is also observed that the pattern repeats with the same periodicity for a long period of time.

Figures 2(a),(c) show during standing and sitting activity, the link quality is good and constant with high PDR. However, depending on the posture of sitting or standing, the quality of link will vary. Sleeping activity can be disrupted when the human rolls towards the bed and there is no direct line-ofsight between the transmitter and receiver. A huge difference in the PDR is seen during the sleeping activity due to the complete shadowing of the RF signals by the body for a significantly longer periods of time, as shown in 2(b).

Finding a time window for such dynamic events, if not impossible, is a complex-process to be implemented on resource constraint IBSN nodes. Idle activities like sleeping can occur with longer duration in the same posture, causing constantly low RSSI and link quality between the nodes. The frequency of the occurrence of human activity is not always predictable and to enable the radio communication only while a favorable link-quality exist is challenging for the MAC protocol design. The time window prediction is useful for activities such as walking and running, in which periodicity is expected, and transmitting only during favorable link quality will ensure high reliability. It is, however, not very useful and energy-efficient in case of idle activities such as sleeping, sitting, or standing.

Use of relay nodes will improve the PDR during idle activities by hopping the information through an intermediate node which is not shadowed by the body posture. Also, use of relay nodes is feasible in IBSN, as the maximum number of hops required is at most two, due to the short distance between the nodes [13]. However, introducing relay nodes will increase the power consumption [13] particularly because of (i) improper design of routing and (ii) unwanted use of relay nodes, which are the same reasons for not recommending the use of relay nodes in MICS band communication by the IEEE 802.15.6 standard [14].

By considering a combination of both physically active and idle activities and their effect on the RSSI, we will design a link quality-aware MAC protocol called the Human ACtivity Medium Access Control (HACMAC). We will follow the regulations (i) set by ETSI for the physical layer parameters of active medical implants and (ii) as suggested by the IEEE 802.15.6 standard for MAC layer parameters for medical implant communication service (MICS). The main contribution of this paper is the design and evaluation of a reliable link quality-aware MAC protocol called HACMAC for implantable body sensor networks which is compliant with the IEEE 802.15.6 MAC regulation.

\section{Human ACtivity Based Medium AcCess CONTROL (HACMAC)}

HACMAC is an hybrid access mechanism based on the IEEE 802.15.6 MAC layer specifications and is intended to operate in MICS band communication [14]. The main aim of the HACMAC is to enhance the reliability of wireless communication when the RF spectrum is distorted by human activities without compromising on the energy-efficiency of the sensor nodes. We assume that the nodes of IBSN are resource-constraint and the nodes $(\mathrm{BN})$ are implanted subcutaneously in the human body with one external body- 


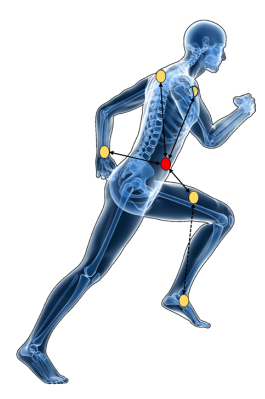

Fig. 4: Location of IBSN nodes in simulation set-up

worn coordinator node (BNC). An example of IBSN is shown in Fig. 4, where the red node is BNC and coffeecolored nodes are implanted subcutaneously in the body. The BN nodes can also act as a relay node (BNR) to hop information with the same mechanism as described in the IEEE 802.15.6 standard [14]. According to this standard, nodes are classified into two categories, namely priority nodes and non-priority nodes.

IEEE 802.15.6 MAC uses super-frame of duration $n$ time units, within which BNC allocates three different phases namely, exclusive access phase (EAP) in which the nodes can only send emergency medical data in contention based fashion, random access phase (RAP) in which all nodes can contend to send medical-data, managed access phase (MAP) in which the BNC allocates either carrier sense multiple access with collision avoidance (CSMA/CA), slotted aloha, polled allocation, or scheduled time division multiple access mechanism (TDMA) for each BN node to send the medical data. The super-frame structure is shown in Fig. 3.

HACMAC is operated in beacon mode, in which a beacon frame $B$ is transmitted from $\mathrm{BNC}$ to the $\mathrm{BN}$ nodes at the start of each super-frame period to facilitate the network management, including the coordination of medium access phases, duty-cycle of the BN nodes connected the BNC, and clock synchronization information. HACMAC uses TDMA during the MAP phase for transmitting the non-emergency data which applies to continuous monitoring of medical symptoms. It uses CSMA/CA during the EAP phase for sending emergency data, which can be an anomaly in the medical data.

It was discussed in section I that the PDR drops substantially depending on the body posture during the activities. This results in poor reliability and energy wastage. HACMAC uses RSSI information and PDR information to detect the human activity and benefits from the periodicity and dynamicity of human activities to improve the reliability and energyefficiency of the wireless communication.

In HACMAC, BNC performs minimalistic operations to adapt the super-frame for reliable communication. BNC does not require additional packets to carry out these operations because the required information, such as RSSI and PDR, are derived from the data-packets transmitted previously. $\mathrm{BNC}$ propagates the adaptive super-frame structure to the $\mathrm{BN}$ nodes through the beacons, which makes HACMAC simple yet powerful. The BN nodes determine their position, link quality, relay mechanism, and periodicity, through the beacon frame of the BNC, based on which the BN node determines the access mechanism, time slot and the relay to transmit the information to BNC.

HACMAC is designed based on two important metrics, i.e.,

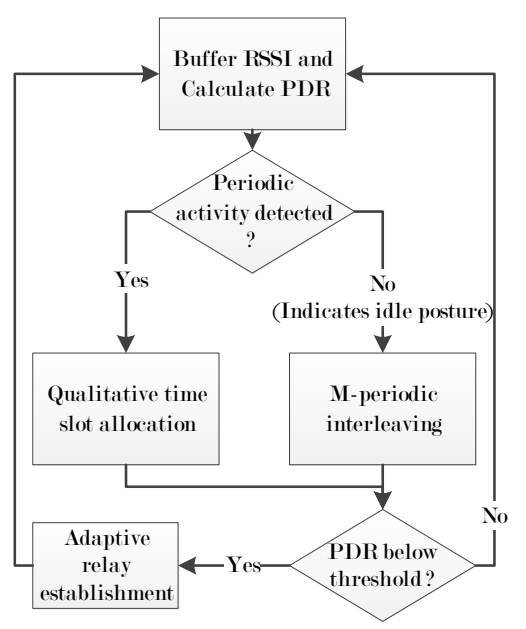

Fig. 5: HACMAC schemes - Flowchart

RSSI and PDR. Although these metrics are noise-prone, careful pre-processing is done to ensure the credibility of these parameters during the run-time of the network.

RSSI sensing function: RSSI sensing function is implemented only on the BNC. Before the network initiation, RSSI values between each $\mathrm{BN}$ node and the $\mathrm{BNC}$ are calibrated and stored in a table, for different activities of a person. The calibration process will identify the mean $\left(\mu=\frac{1}{N} \sum_{i=1}^{N} x_{i}\right)$ and standard deviation $(\sigma=$ $\sqrt{\left.\frac{1}{N} \sum_{i=1}^{N}\left(x_{i}-\mu\right)^{2}\right)}$ of RSSI, for different activities, which are used for simple rule-based classification of activities. Since the location of nodes are fixed in IBSN, the calibration remains valid for longer periods of time. Periodicity of the activity is determined by peak-detection using mean crossing rate and deriving the time stamps for each of the RSSI values which crosses a threshold of the mean value. Upon network initiation, the RSSI sensing function will buffer RSSI after each super-frame period, as per the requirements of HACMAC schemes.

PDR sensing function: BNC calculates PDR using number of successful reception of packets within minimum number of re-tries. We use the acknowledgment scheme of IEEE 802.15.6 standard to calculate the PDR by sensing the sequence number and the acknowledgement of a packet, which is similar to the Expected Number of Transmissions (ETX) [18] method of estimating the link quality.

HACMAC uses three main schemes, namely, (i) qualitative time-slot allocation, (ii) m-period interleaving, and (iii) adaptive relay establishment to function robust against RF shadowing caused by the human activities irrespective of their dynamicity and non-periodicity. In what follows we elaborate on each of these three schemes.

\section{A. Qualitative time-slots allocation}

As shown in Figure 2(d) and Figure 2(e), the periodic activities performed by human beings have sufficient time periods of favorable link quality. On the BNC node, RSSI values of the $\mathrm{BN}$ nodes are buffered for $T_{n}$ time period in a buffer of $n_{b u f}$, after each super-frame period $n$. As soon as the buffer is full, the BNC node calculates the mean and variance of the RSSI values, which are unique for each periodic activity. Average periodicity of each activity is 
calibrated for a specific person and pre-stored on the BNC. The BNC adapts the time-slot allocated to the BN node such that the node transmits only when favorable RSSI is sensed. By doing so, even if the RSSI value is lowered down over time because the person moves in different environments while performing different activities, the signal to noise ratio will still be high when the link between the BN and the BNC nodes is not shadowed by the human body. Such high signal to noise ratio will result in a good PDR.

\section{B. M-periodic interleaving}

If the RSSI values do not exhibit any periodicity, the $\mathrm{BNC}$ assumes that the person is idle and distributes the time slot equally between all BN nodes. However, a person can be static in a body posture, but the link between a BN node and BNC maybe shadowed. We will use the RSSI sensing function of the BNC explained before to allocate time slots for the BN nodes such that for the BNs node with the highest shadowing, additional redundant time-slots will be allocated. This time-slot allocation will be constant for a period of $m$ super-frames until the RSSI sensing function detects periodicity.

Sequentially, the PDR sensing function will accumulate packet delivery information for all $\mathrm{BN}$ nodes. If PDR of a specific $\mathrm{BN}$ node decreases during the m-periodic interleaving, a relay node (BNR) between the BNC and the BN has to be instantiated to relay the packet to guarantee the required PDR. If a relay node is instantiated, the adaptive time slot allocation is reset immediately, reverting back to the equally distributed time-slot allocation.

\section{Adaptive relay establishment}

As explained above, the prolonged shadowing effect of RF link between the $\mathrm{BN}$ and the $\mathrm{BNC}$ nodes can be improved by selecting a relay node between them. The mechanism of connecting the relay node in HACMAC is inspired by the IEEE 802.15.6 standard [14].

Prolonged shadowing of the BN increases the possibility of missing life-critical medical events and also may increase the latency. As the transmission power cannot be increased in the MICS RF band, temporary use of relay nodes is an optimal solution to overcome this situation. Moreover, in HACMAC the relay node is used only when a low PDR is detected and is disabled otherwise. This reduces the unnecessary wastage of energy on the $\mathrm{BN}$ node.

The location of nodes in IBSN is fixed once implanted, which makes the selection of relay nodes simple. Time-slot of the relay node will be compensated by an additional timeslot allocation in which the relay node can transmit its own data. The RSSI and PDR sensing functions of the BNC will decide about the duration for which the relay node has to function.

\section{Operation of $B N$ and $B N R$}

The $\mathrm{BN}$ node receives the beacons and identifies the duration of each phase and the time slot allocated for it. If there is no data to be sent, the $\mathrm{BN}$ node transmits the no-data frame and goes to sleep, otherwise it transmits on the time slot allocated to it. In case of a emergency event or an anomaly in the sensed medical data, the BN node

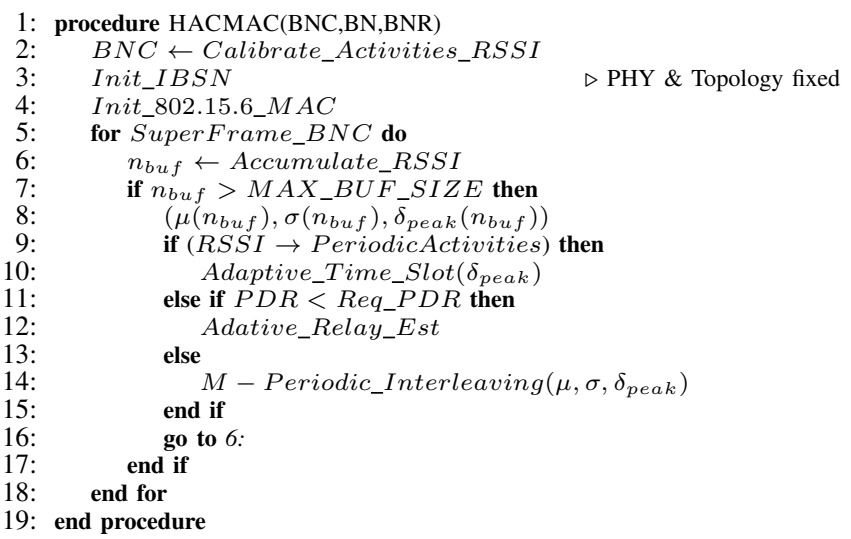

\section{Fig. 6: Pseudocode of HACMAC operation}

contends to send data in the consecutive EAP phase with reference to the latest received beacon. BN node does not have to send any additional packets for the operation of HACMAC.

In case of the relay initiation, $\mathrm{BN}$ node which acts as the BNR will encapsulate the data in its frame and transmit it to the BNC in the additional time-slot allocated for it. This process is similar to the relay mechanism as described in IEEE 802.15.6 standard [14]. The choice of selecting a $\mathrm{BN}$ node as a relay node is pre-determined based on the location of the node. For example, in Fig.4, the BN node in the ankle, uses BN node in the thigh as the relay node. Since the location of these two nodes is fixed, the choice of relay node is easily pre-determined and the relay-topology for each $\mathrm{BN}$ node is pre-stored in the BNC.

\section{EvaluATION OF HACMAC}

For the purpose of evaluation, we use simulations in an OMNeT++ based simulator called Castalia v3.2 [19]. Castalia simulator has good implementation of temporal variations of the physical channel and is modifiable in the simulator. We measured the RSSI values between each BN node and the BNC in a round-robin fashion, during each activity in an indoor environment. We model 5 different activities namely, sitting, standing, sleeping, walking and running, since these activities cover both the periodic and non-periodic activities. The $\mathrm{BN}$ nodes were sending a dummy packet of 40 bytes to the BNC every $10 \mathrm{~ms}$ (optimal for measuring the impact of human activities in RSSI [13]), with which the BNC calculates the RSSI and PDR, while the human was performing each activity. These RSSI values are translated to temporal variations as mentioned in [19]. In addition to our measured data, we also used the data from [13] and [10] to simulate the temporal variations of the physical channel caused due to the human activities.

For the simulation of MAC protocols we used the PHY parameters as listed in Table I. We simulated $5 \mathrm{BN}$ nodes which are located in two limbs, thigh, chest and back-shoulder, and one BNC connected in a star topology, as shown in Figure 4, which is suitable for most of the medical applications [12]. Note that the thigh node is a potential relay node between the ankle $\mathrm{BN}$ node and the $\mathrm{BNC}$. We vary the data generation rate for each node in order to simulate the heterogeneity of the IBSN nodes that generate the medical data at different rates, for example 
a blood-glucose sensor and a heart rate sensor. In order to compare the performance HACMAC with existing MAC protocols, we simulate IEEE 802.15.6 MAC without wake-up radio [14], BANMAC [16] which uses OTW to access the wireless medium, and BATMAC [17], which uses relay nodes to transmit data with variable super-frame period, in the same physical channel described in [12]. We use specifications of the Microsemi ZL70102 radio [20] to evaluate the power consumption of the BNC, BN, and BNR nodes. However, it has to be noted that the actual power consumption depends on the hardware design of the sensor nodes and the overheads caused by the additional hardware to support the functioning of the wireless radio.

\begin{tabular}{lrl}
\hline Parameter & \multicolumn{2}{c}{ Value } \\
\hline Channel bandwidth & 300 & $\mathrm{KHz}$ \\
Reception current & 7 & $\mathrm{~mA}$ \\
Transmission current (main) & 11 & $\mathrm{~mA}$ \\
Idle current (main) & 3 & $\mathrm{~mA}$ \\
Sleep current & 3 & $\mu \mathrm{A}$ \\
Slot time (TDMA) & 500 & $\mu \mathrm{s}$ \\
Number of retries (CSMA) & 10 & - \\
Goodput & 40 & $\mathrm{Kbps}$ \\
\hline
\end{tabular}

TABLE I: Simulation parameters

\section{RESUlts}

Simulation results related to reliability and packet loss are shown in Figure 7-11. Figure 7 illustrates the average PDR of the network calculated on the BNC node. The IEEE 802.15.6 MAC without wake-up radio has the lowest success in terms of packet-delivery ratio because of lack of any mechanism to compensate for the high attenuation caused by the human activities. At high data generation rates, the IEEE 802.15.6 MAC has around 20\% packet loss. This indicates, the need for a mechanism which can compensate for the attenuation caused by the human activities. BANMAC protocol predicts OTW to compensate for the periodic activities like walking and running, hence it outperforms the IEEE 802.15.6 MAC at higher data generation rates. However, it still has $15 \%$ packet loss at higher data rates, which is not desirable for IBSN. Such high packet loss is due to the lack of any mechanism to compensate for the prolonged attenuation caused by idle activities such as sleeping and sitting. BATMAC uses relay nodes to compensate for long attenuation but it cannot compensate for high attenuation caused by periodic activities. BATMAC has the worst performance with almost $25 \%$ packet loss because it does not take into account the short periods of favorable links at higher data generation rates. In contrary, HACMAC has the lowest packet loss of $10 \%$ even at higher data generation rates, due to the fact that it compensates for the prolonged attenuation caused by idle activities and benefits from the shorter periods of favorable link quality to achieve better performance in all the scenarios.

Application level latency is the difference in time at which the packet is generated and the time at which packet is received at the receiver. BATMAC which uses purely time-slot allocation with adaptive super-frame structure has more delay when more packets are generated. On an average, the hybrid MAC protocols, IEEE 802.15.6, BATMAC, and HACMAC have uniform distribution of

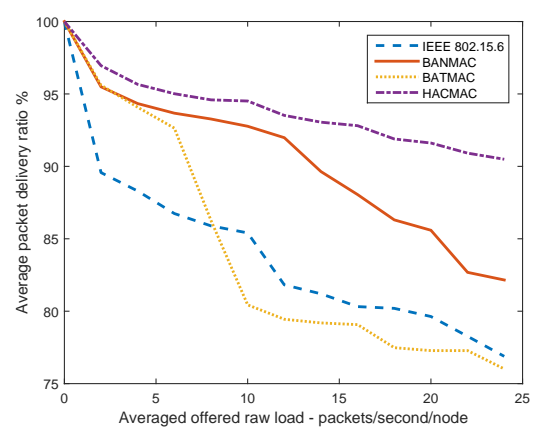

Fig. 7: Offered load vs Packet delivery ratio

latency, which is due to its uniform super-frame duration. OTW prediction in BANMAC adds overhead packets to be transmitted which significantly increases the latency of successful communication. IEEE 802.15.6 MAC has no such mechanism but still suffers from temporal variations in the wireless channel, which results in a slightly higher latency compared to HACMAC. HACMAC has pre-stored information, which can overcome temporal variations in the channel and perform better in latency than the rest of the MAC protocols

The power consumption evaluation results of the MAC protocols are demonstrated in Fig. 9- 11. The power consumed is calculated by measuring the periods at which the $\mathrm{BNC}, \mathrm{BN}$ and $\mathrm{BNR}$ are transmitting, receiving, idly listening (radio is turned on but not in receive mode) or sleeping (radio is turned off). Note that, each activity has different power consumption as mentioned in Table. I. The power consumption of the BNC is vastly reduced in HACMAC due to the fact that it does not listen for a long period and transmits no extra beacons to calculate the PDR. HACMAC benefits from the characteristics of the human activities and pre-predicting the activity and its attenuation characteristics. On the other hand, BANMAC requires additional receiving and transmitting periods to effectively calculate the OTW. BATMAC suffers from repeated transmissions to decide the super-frame period and the selection of the relay nodes. It wastes extra power to decide on the relay nodes when the topology is fixed, which results in additional power consumption at higher data generations rates. The IEEE 802.15.6 MAC has no mechanism to compensate for the attenuation at all, which results in higher number of retries in prolonged attenuation periods, than the rest of the MAC protocols. The difference between power saving mechanisms in HACMAC and BATMAC on the BN nodes is quite small, because both of them use similar process in selecting the relay nodes. The only difference between the two MAC protocols is, in HACMAC the BN nodes are excluded from the process of deciding the relay nodes in longer attenuation periods. In BANMAC, the BN nodes have higher power consumption due to the additional reception and transmission required for the OTW prediction. In the IEEE 802.15.6 MAC, the $\mathrm{BN}$ nodes have the highest power consumption since they suffer from higher number of re-transmissions to ensure reliability.

BANMAC has no relay mechanism to compare the power consumption of the relay nodes. We used BATMAC and IEEE 802.15.6 MAC with 1 hop extended star-topology to compare their power consumption with HACMAC. In 


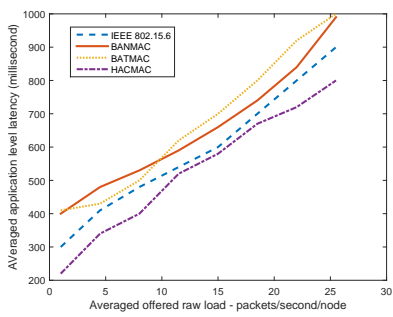

Fig. 8: Offered load vs Application level latency of IBSN

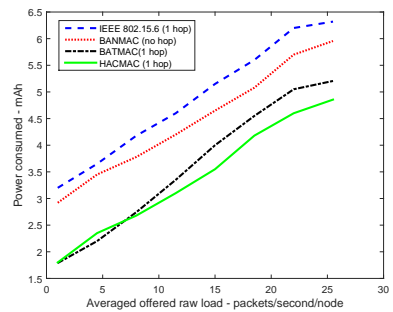

Fig. 10: Power consumption BN

BATMAC, the BNR nodes continuously listen for the packets and re-route the packets depending on the link quality to the BNC. This process requires the BNR to be always in the receive mode when it is not transmitting, making the BNR power hungry. To improve this process, in HACMAC the BNC selects the BNR nodes by calculating the link quality after each super-frame duration which enables the BNR nodes to be in idle mode in most of the super-frame duration, and BNR enters the receive mode only in the scheduled time slot. Energy-efficiency of HACMAC over BATMAC is realized in higher packet generation rates as shown in Fig. 11. In the IEEE 802.15.6 MAC, the BNR nodes try to poll the source BN node for any remaining data and has no pre-stored information about the topology, which results in additional packets to be sent between BNR and BNC to make an agreement in relay mechanism. These additional packets can consume extra power when the channel is dynamically varied in link quality and higher number of retries are required for a successful reception of the packet.

\section{CONCLUSION}

We presented the HACMAC protocol, which complies with the IEEE 802.15.6 regulations and adapts dynamically to different human activities in order to guarantee a high reliability of wireless communication being distorted by shadowing effect. We compared the performance of HACMAC in terms of packet loss through simulation and demonstrated the need to handle dynamic attenuation caused by different kinds of human-activities. Power consumption of the wireless radio decreased greatly with HACMAC when compared to the IEEE 802.15.6 MAC, BANMAC, and BATMAC.

\section{ACKNOWLEDGMENT}

This research is funded by STW project Cyber Physical Systems (CPS).

\section{REFERENCES}

[1] Z. Shlegr and A. Egorov, "Implantable electric bladder stimulator used for neurogenic failures," Biomedical Engineering, vol. 7, no. 6, pp. 382-383.

[2] K. Rajappan, "Permanent pacemaker implantation technique: part ii," Heart, vol. 95, no. 4, pp. 334-342, 2009.

[3] K. Hutchinson and J. Y. Wick, "Deep brain stimulation and medication management in parkinson's disease," The Consultant Pharmacist, vol. 31, no. 2, pp. 73-84, 2016.

[4] A. Alwan et al., Global status report on noncommunicable diseases 2014. World Health Organization, 2016. [Online]. Available: http://www.who.int/nmh/publications/ncd-status-report-2014/en/

[5] K. Bazaka and M. V. Jacob, "Implantable devices: Issues and challenges," Electronics, vol. 2, no. 1, p. 1, 2013. [Online]. Available: http://www.mdpi.com/2079-9292/2/1/1

[6] J. Yeun-Ho, "Development of implantable medical devices: From an engineering perspective," Int Neurourol J, vol. 17, no. 3, pp. 98-106, 2013.

[7] S. Ramgopal, S. Thome-Souza, M. Jackson, N. E. Kadish, I. S Fernández, J. Klehm, W. Bosl, C. Reinsberger, S. Schachter, and T. Loddenkemper, "Seizure detection, seizure prediction, and closedloop warning systems in epilepsy," Epilepsy \& Behavior, vol. 37, pp. 291-307, 2014.

[8] V. Chaganti, D. Smith, and L. Hanlen, "Second order statistics for many-link body area networks," in IEEE Antennas and Wireless Propagation Letters., apr 2010, pp. 322-325.

[9] K. S. Prabh and J.-H. Hauer, "Opportunistic packet scheduling in body area networks," in Wireless Sensor Networks. Springer, 2011, pp. 114-129.

[10] R. D'Errico and L. Ouvry, "Time-variant ban channel characterization," in Personal, Indoor and Mobile Radio Communications, 2009 IEEE 20th International Symposium on. IEEE, 2009, pp. 30003004.

[11] P. W. Lee, W. K. Seah, H.-P. Tan, and Z. Yao, "Wireless sensing without sensorsan experimental approach," in Personal, Indoor and Mobile Radio Communications, 2009 IEEE 20th International Symposium on. IEEE, 2009, pp. 62-66.

[12] V. R. K. Ramachandran, B. J. van der Zwaag, N. Meratnia, and P. J. Havinga, "Evaluation of MAC protocols with wake-up radio for implantable body sensor networks," Procedia Computer Science, vol. 40, pp. 173 - 180, 2014, fourth International Conference on Selected Topics in Mobile \& Wireless Networking (MoWNet 2014).

[13] F. Di Franco, I. Tinnirello, and Y. Ge, "1 hop or 2 hops: Topology analysis in body area network," in Networks and Communications (EuCNC), 2014 European Conference on. IEEE, 2014, pp. 1-5.

[14] "Ieee standard for local and metropolitan area networks - part 15.6: Wireless body area networks," Feb 2012, pp. 1-271.

[15] K. Levis, "Rssi is under appreciated," in Proceedings of the Third Workshop on Embedded Networked Sensors, Cambridge, MA, USA, vol. 3031, 2006, p. 239242.

[16] K. S. Prabh, F. Royo, S. Tennina, and T. Olivares, "Banmac: an opportunistic mac protocol for reliable communications in body area networks," in Distributed Computing in Sensor Systems (DCOSS), 2012 IEEE 8th International Conference on. IEEE, 2012, pp. 166175.

[17] M. Maman and L. Ouvry, "Batmac: an adaptive tdma mac for body area networks performed with a space-time dependent channel model," in Medical Information \& Communication Technology (ISMICT), 2011 5th International Symposium on. IEEE, 2011, pp. $1-5$.

[18] D. S. J. De Couto, D. Aguayo, J. Bicket, and R. Morris, "A high-throughput path metric for multi-hop wireless routing," in Proceedings of the 9th Annual International Conference on Mobile Computing and Networking, ser. MobiCom '03. New York, NY, USA: ACM, 2003, pp. 134-146.

[19] NICTA, "Castalia simulator," Accessed February 2016. [Online]. Available: http://castalia.npc.nicta.com.au

[20] Microsemi, "Z170102 full datasheet," Accessed February 2016. [Online]. Available: http://www.microsemi.com 